\title{
The effect of stoma education class on peristomal dermatitis in colorectal cancer patients with defunctioning ileostomy-a retrospective study of 491 patients
}

\author{
Dandan He ${ }^{1,2 \#}$, Weiwen Liang ${ }^{1,2 \#}$, Qiuqiong Yao ${ }^{1,2}$, Jie Zhao ${ }^{1,2}$, Rang Liu ${ }^{1,2}$, Guiting Chen ${ }^{1,2}$, \\ Huaiming Wang ${ }^{1,2}$, Xinmei Ye ${ }^{1,2}$, Rongkang Huang ${ }^{1,2}$ \\ ${ }^{1}$ Department of Colorectal Surgery, the Sixth Affiliated Hospital, Sun Yat-sen University, Guangzhou, China; ${ }^{2}$ Guangdong Provincial Key \\ Laboratory of Colorectal and Pelvic Floor Diseases, the Sixth Affiliated Hospital, Sun Yat-sen University, Guangzhou, China \\ Contributions: (I) Conception and design: H Wang, X Ye, R Huang; (II) Administrative support: H Wang, R Huang; (III) Provision of study materials \\ or patients: All authors; (IV) Collection and assembly of data: All authors; (V) Data analysis and interpretation: D He, W Liang, R Huang; (VI) \\ Manuscript writing: All authors; (VII) Final approval of manuscript: All authors. \\ \#These authors contributed equally to this work. \\ Correspondence to: Huaiming Wang, PhD. Department of Colorectal Surgery, the Sixth Affiliated Hospital, Sun Yat-sen University, Guangzhou, \\ China. Email: wanghm7@mail.sysu.edu.cn; Xinmei Ye. Nursing Department, the Sixth Affiliated Hospital, Sun Yat-sen University, Guangzhou, \\ China. Email: sanfanzhou@126.com; Rongkang Huang. Department of Colorectal Surgery, the Sixth Affiliated Hospital, Sun Yat-sen University, \\ Guangzhou, China. Email: huangrk3@mail.sysu.edu.cn.
}

Background: Ileostomy was widely used during colorectal cancer (CRC) surgery and peristomal dermatitis was one of the most common stoma-relative complications. Stoma education class may reduce the rate of peristomal dermatitis.

Methods: We enrolled patients who were diagnosed with rectal cancer and underwent surgery with ileostomy between January 2018 to December 2018 at a single tertiary hospital. The general demographic information of patients along with the participation in stoma education class and the occurrence of peristomal dermatitis were analyzed.

Results: A total of 491 patients were included in the study, and 162 patients $(32.99 \%)$ participated in the stoma education class. Eighty-five patients (17.31\%) suffered peristomal dermatitis within one month after ileostomy. The rate of peristomal dermatitis in the stoma education group was significantly lower than that in the control group (11.11\% vs. 20.36\%, $\mathrm{P}=0.011)$. Regardless of the education level, the risk of peristomal dermatitis in the education group was reduced $(\mathrm{P}<0.05)$. Lower peristomal dermatitis rates were found in patients who were younger than 60 years $(\mathrm{P}=0.012)$, whose stoma were taken care of by other people $(\mathrm{P}=0.014)$, or without diabetes $(\mathrm{P}=0.026)$. Univariate and Multivariate analysis showed that stoma education was the only factor associated with the decrease in rates of peristomal dermatitis ( $O R=0.458, P=0.008)$, while diabetes was an independent risk factor $(\mathrm{OR}=3.732, \mathrm{P}<0.001)$.

Conclusions: Postoperative stoma education class significantly decreased the rate of peristomal dermatitis in the early postoperative period in CRC patients with ileostomy, especially for those who were younger than 60 years, received stoma care from others or without diabetes.

Keywords: Stoma education; peristomal dermatitis; ileostomy; colorectal cancer (CRC)

Submitted Nov 15, 2020. Accepted for publication Dec 06, 2021.

doi: $10.21037 /$ tcr-20-3267

View this article at: http://dx.doi.org/10.21037/tcr-20-3267 


\section{Introduction}

Colorectal cancer (CRC) is the third leading cause of cancer worldwide (1). Defunctioning loop ileostomy is selectively performed during CRC surgery. Although ileostomy cannot prevent leakage itself, a defunctioning ileostomy can minimize the aggravation of clinical symptoms (2). However, the formation of ileostomy could have a great negative impact on the quality of life, including electrolyte disturbances, readmission, increased costs and peristomal dermatitis (3). New stoma patients face significant physical, psychological and body image adaption due to the lack of information with ileostomy care $(4,5)$. Patients often rely heavily on the assistance of specialized stoma care nurses during their hospital stay rather than independently managing their own stoma. Specialized guidance and expertise can improve the quality of stoma-care and problem-solving abilities for new stoma patients $(6,7)$. Patient education was reported to have a positive impact on self-management skills (8).

Peristomal dermatitis is the most common stoma-related complication (9), especially when an ileostomy is present in CRC patients. There are few studies of the influence of stoma education on early stoma complications such as dermatitis. Hignett et al. (10) reported that strengthening the health education of stoma can effectively reduce the stoma complications. Stokes et al. (11) also confirmed that a preoperative stoma education group class significantly reduced the likelihood of peristomal dermatitis. So far there has been little agreement on the role of stoma education, while some studies reported negative results, which suggested that perioperative stoma education has no effect on the stoma complications $(7,12)$.

There is a continued need for further study to test and verify the influence of stoma education on the risk of peristomal dermatitis. A postoperative stoma education class has been held by the stoma team in our institution for a decade, and a retrospective study of 491 CRC patients with ileostomy was carried out. We postulate that well-educated patients will have a lower risk of peristomal dermatitis in the early postoperative period compared to patients who did not participate in the stoma education class. We present the following article in accordance with the STROBE reporting checklist (available at http://dx.doi.org/10.21037/tcr-20-3267).

\section{Methods}

\section{Patients}

Our study collected the data of CRC patients who underwent surgery with ileostomy at the Sixth Affiliated Hospital of Sun Yat-sen University from January 2018 to December 2018. Peristomal dermatitis was assessed during the outpatient visit within one month after surgery. And the general demographic information of patients along with the participation in stoma education class and the occurrence of peristomal dermatitis were analyzed.

CRC Patients older than 18 years who underwent ileostomy were eligible for inclusion, and patients with previous abdominal surgery, emergency and/or urgent surgery or who had incomplete or missing follow-up data were excluded.

The study was conducted in accordance with the Declaration of Helsinki (as revised in 2013). The study was approved by the Ethics Committee of the Sixth Affiliated Hospital of Sun Yat-sen University (NO.2020ZSLYEC-040). Informed consent is not needed in this observational and retrospective study.

\section{Criteria of peristomal dermatitis $(13,14)$}

Peristomal dermatitis is the development of erythema, edema, possible vesicles, maceration and loss of skin integrity as a result of contact with a number of substances including urine, feces, medicaments, ostomy pouch systems, and stoma skin care products, and the common forms of peristomal dermatitis include irritant contact dermatitis, mechanical dermatitis, and allergic contact dermatitis.

\section{Content of stoma education class}

The stoma education class refers to the theoretical training of stoma nursing held by the stoma team in our research institute every two weeks. The speakers are all certified nurses, and patients, along with their spouses/partners/ caregivers were invited to attend the one-hour education within 2 weeks after ileostomy operation. A PowerPoint presentation was prepared that included assessment of the stoma and peristomal skin, assessment of stoma output, stoma-related supplies and resources, nutrition and fluids, activities of daily living, sports and work. Patients and their caregivers were encouraged to both ask questions and share their prior knowledge regarding stoma-related issues to correct inaccurate information. At least one stoma volunteer with more than 5 years of stoma-care experience was invited to the following group discussion to share their experience and to alleviate any exaggerated concerns. Patients and their partners or caregivers were given a hands-on booklet 
regarding care of ileostomy after class. All patients were shown how to and practiced emptying and changing their pouch at least 2-3 times before the day of discharge by the ward colorectal nurse specialist.

\section{Data collection}

The data of patients were obtained through clinics and telephone follow-up. The researchers extracted the followup information of CRC patients with ileostomy from January to December 2018, and the follow-up time was up to January 30, 2019.

In total, 15 explanatory variables were recorded. These included information on demographics, indications, comorbid and surgical factors: gender, age, marital status, education level, caregiver, smoking status, diabetes, defecation condition, defecation volume, stoma type, preoperative marking, radiotherapy, chemotherapy, stoma class and peristomal dermatitis.

\section{Statistical analysis}

Statistical analysis was performed using IBM SPSS Statistics version 25.0. Descriptive statistical methods were performed to characterize the patients and data were presented as median and range. Chi-square tests was used in univariate and stratified analysis was made for the age group ( $\leq 60$ and $>60)$ and caregiver and diabetes group. Logistic regression models were fitted to calculate OR value and $95 \%$ CI of the occurrence of peristomal dermatitis associated with all covariates with a $\mathrm{P}$ value of 0.05 or less in univariate comparisons. Differences were considered statistically significant when $\mathrm{P}<0.05$.

\section{Results}

A total of 491 patients were included in the study, and 162 patients (32.99\%) participated in the one-hour stoma education class within 2 weeks after ileostomy operation. A total of 85 patients $(17.31 \%)$ suffered peristomal dermatitis within one month after ileostomy. The rate of peristomal dermatitis in the stoma education group was significantly lower than that in the control group $(11.11 \%$ vs. $20.36 \%$, $\mathrm{P}=0.011$, Table 1). Regardless of the education level, the risk of peristomal dermatitis in the stoma education class was reduced $(\mathrm{P}<0.05)$. Stratified by age $(\leq 60$ and $>60)$, caregiver (self-care and others-care) and diabetes, significant lower peristomal dermatitis rates were found in patients who were younger than 60 years $(\mathrm{P}=0.012)$, whose stoma was cared for by other people $(\mathrm{P}=0.014)$, or without diabetes $(\mathrm{P}=0.026)$ in further stratified analysis (Table 2).

Univariate analysis was performed to estimate the related factors of occurrence of peristomal dermatitis. The results showed that gender $(\mathrm{P}=0.031)$, diabetes $(\mathrm{P}=0.001)$, and stoma class $(\mathrm{P}=0.011)$ were significant factors in the occurrence of peristomal dermatitis (Table 3). Logistic regression was then used to identify the effects of the covariates on the occurrence of peristomal dermatitis and showed that the stoma education class was the only significantly factor associated with the decrease in the rate of peristomal dermatitis $(\mathrm{OR}=0.458, \mathrm{P}=0.008)$, while diabetes was an independent risk factor $(\mathrm{OR}=3.732$, $\mathrm{P}=0.001$; Table 4).

\section{Discussion}

The most common type of stoma complications are peristomal skin problems, retraction, hernia, prolapse and necrosis (15), which are divided into early stage complications and late stage complications according to whether they occurred within one month or not (16). The rates of stoma complications have been reported to range from $10 \%$ to $72 \%$, and the rates of peristomal dermatitis were $12 \%$ to $62 \%$ (16-18). In our study, the rates of peristomal dermatitis was $17.31 \%$ in the first 30 days after ileostomy creation. Leakage is a critical factor in the development of peristomal skin complications (19). Inadequate consideration of appliance leakage is of particular significance to patients at times of deep distress and a lack of practical knowledge and skill hinders patients' mastery of stoma self-care (20). Stoma nurse specialists should pay more attention to teaching patients the knowledge of stoma care and stoma products (21).

Enhanced recovery after surgery (ERAS) care is well established in colorectal surgery in decade years, and is associated with a reduction in length of stay and morbidity rates $(22,23)$. A certain number of patients with stoma failed to receive adequate guidance of stoma nursing during hospitalization. An educational program was recommended to increase the knowledge of stoma-care, resulting in improved stoma self-management for new stoma patients $(3,6,24,25)$. To solve similar problems, under the premise of ensuring that every ostomate receives 2-3 rounds of stoma care skills guidance during hospitalization, our stoma team holds centralized health education for ostomate regularly. It was found that the incidence of peristomal dermatitis in patients 
Table 1 Patients' sociodemographic, clinical characteristics between the control group and the stoma education group

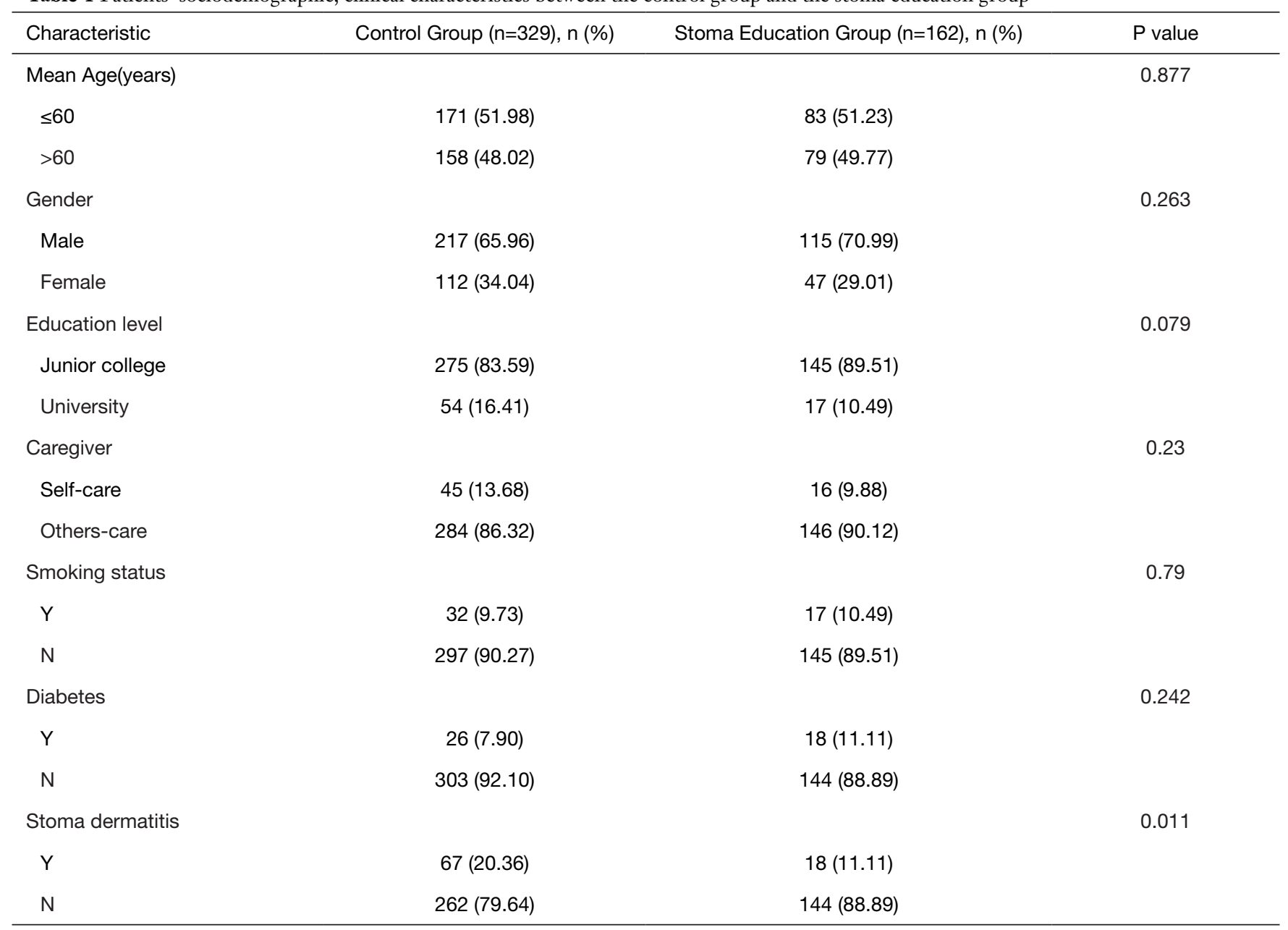

who did not participate in the stoma education class was significantly higher $(20.36 \%$ vs. $11.11 \%, \mathrm{P}=0.011)$ in our study. Multivariate analysis confirmed that the stoma education class was an independent protective factor $(\mathrm{OR}=0.458, \mathrm{P}=0.008)$. The results are consistent with the study of Stokes et al. (11), which confirmed that the rate of peristomal dermatitis of patients who participated in a preoperative 2 -hour stoma education class was significantly lower. Preoperative education tends to explain the concept of stoma and make preparation such as stoma marking before the stoma operation, while postoperative education pay attention to the nursing knowledge and skills for stoma. What's more, they complement each other and are beneficial to reduce the peristomal dermal complications. And our institution has carried out preoperative stoma site and preoperative stoma education and we will try to form a systematic and standardized education course in the future.

The factors related to ostomy complications were gender, age, diabetes mellitus and preoperative stoma site marking $(7,17,26)$. Univariate analysis in our study suggested that gender was an independent factor for peristomal dermatitis $(\mathrm{P}=0.031)$, and females were more likely to suffering peristomal dermatitis. However, logistic regressions analysis showed female tended to show increased occurrence of peristomal dermatitis but not reach statistical significance. There was not clear relation between gender and occurrence of peristomal dermatitis (27).

Park et al. (16) found that elderly patients with stoma are more likely to have complications, but in our study, there was no significant difference in the incidence of peristomal dermatitis in patients with different ages. Stratified analysis showed that, for those who were younger than 60 years old, the rates of peristomal dermatitis were significantly lower 
Table 2 Association between peristomal dermatitis and stoma education class, stratified by age group, education level, caregiver or diabetes group

\begin{tabular}{|c|c|c|c|c|c|}
\hline \multirow{2}{*}{ Characteristic } & \multicolumn{2}{|c|}{ Stoma Education Group } & \multicolumn{2}{|c|}{ Control Group } & \multirow{2}{*}{$P$ value } \\
\hline & RPD, n (\%) & OR $(95 \% \mathrm{Cl})$ & RPD, n (\%) & OR $(95 \% \mathrm{Cl})$ & \\
\hline \multicolumn{6}{|l|}{ Mean age (years) } \\
\hline$\leq 60$ & $7(8.43)$ & $2.496(1.160-5.370)$ & $36(21.05)$ & $0.862(0.779-0.954)$ & 0.012 \\
\hline$>60$ & $11(13.92)$ & 1.409 (0.749-2.653) & $31(19.62)$ & $0.934(0.830-1.050)$ & 0.279 \\
\hline Junior college & $18(12.41)$ & 1.640 (1.003-2.682) & $56(20.36)$ & $0.909(0.835-0.990)$ & 0.042 \\
\hline University & $0(0.00)$ & - & $11(20.37)$ & $0.796(0.696-0.911)$ & 0.043 \\
\hline \multicolumn{6}{|l|}{ Caregiver } \\
\hline Self-care & $2(12.50)$ & $1.600(0.386-6.633)$ & $9(20.00)$ & $0.914(0.722-1.158)$ & 0.503 \\
\hline $\mathrm{N}$ & $14(9.72)$ & 1.833 (1.054-3.188) & $54(17.82)$ & $0.910(0.845-0.981)$ & 0.026 \\
\hline
\end{tabular}

*, stoma care from other people. RPD, rate of peristomal dermatitis.

Table 3 Univariate analysis for occurrence of peristomal dermatitis

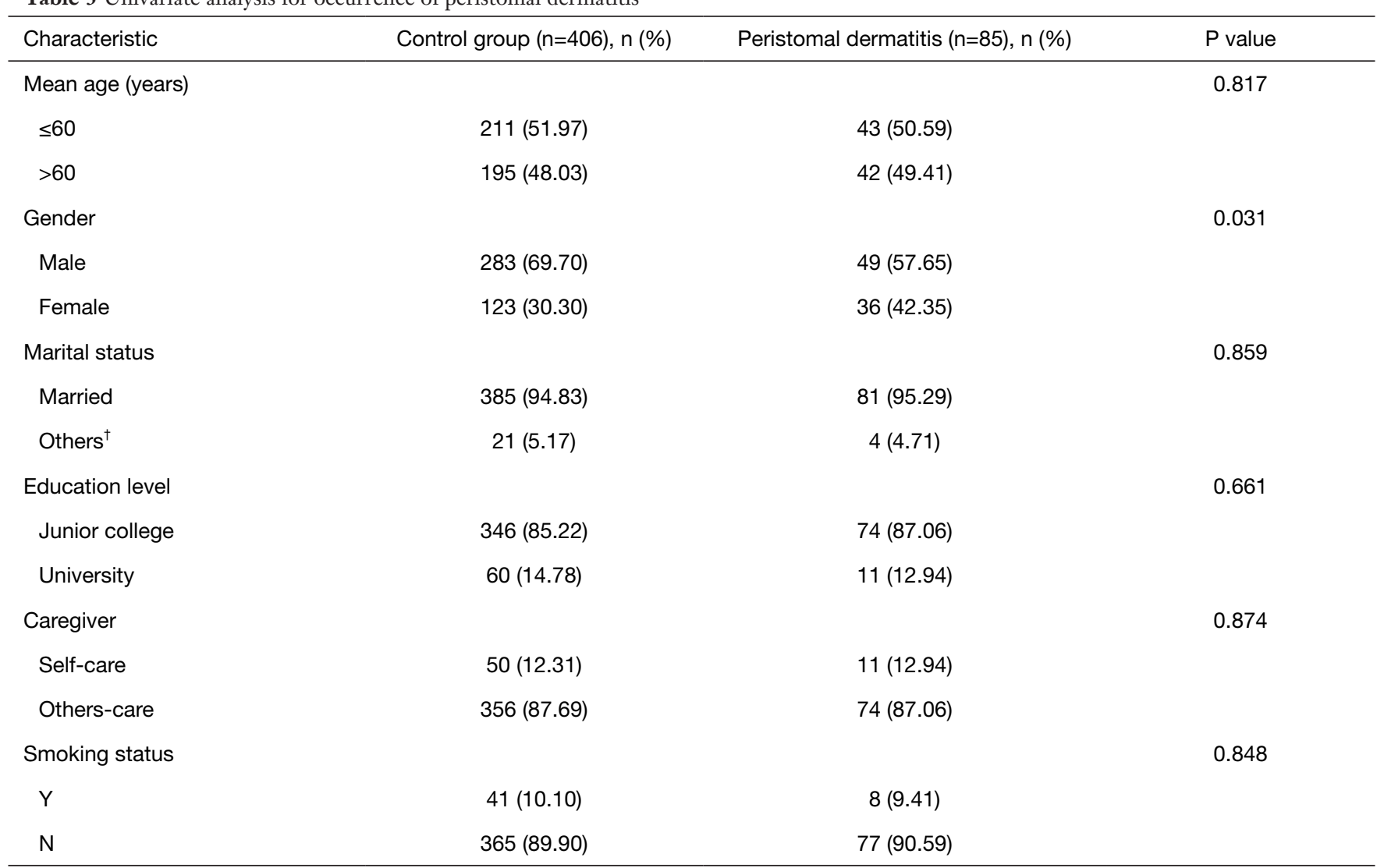

Table 3 (continued) 
Table 3 (continued)

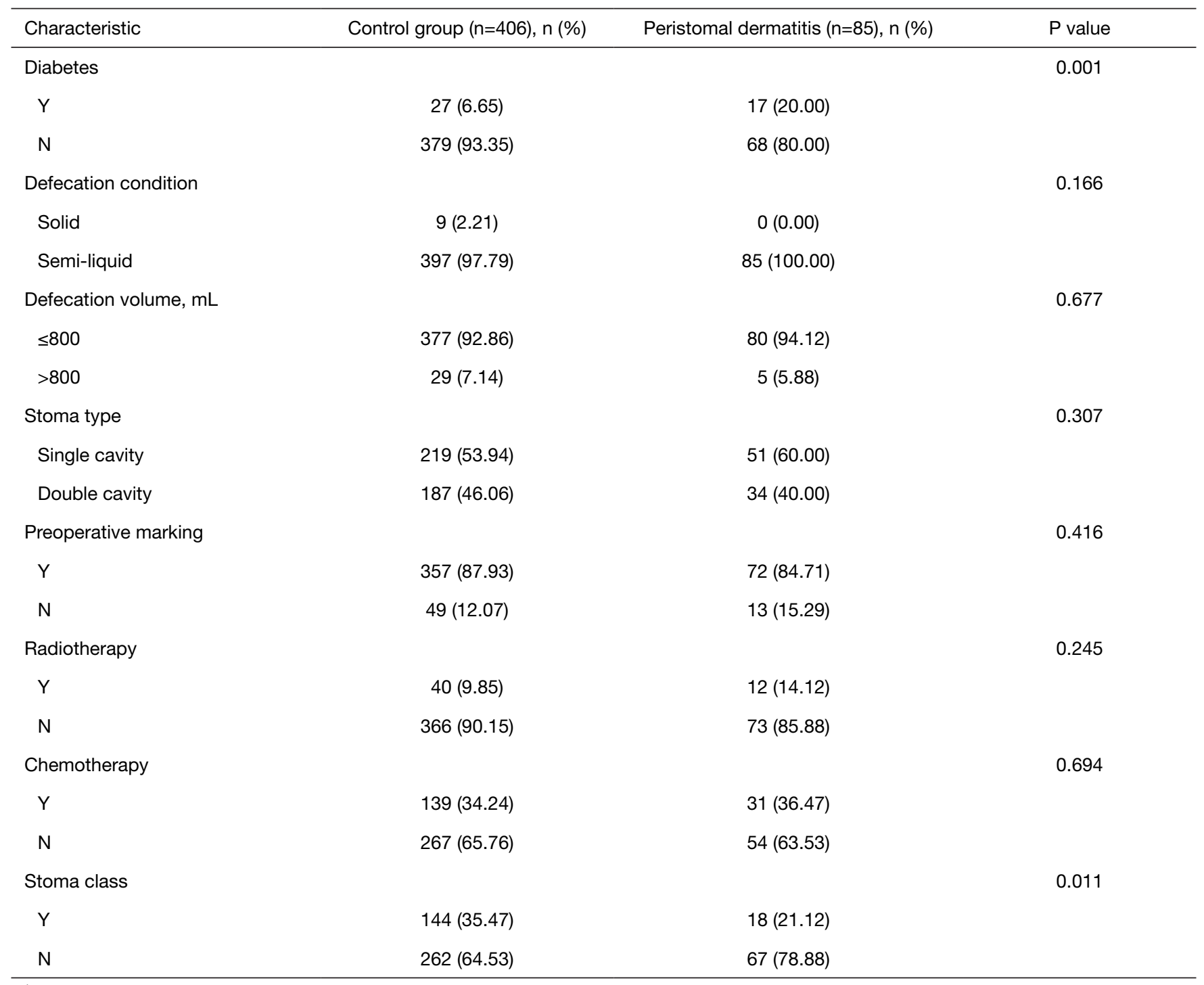

${ }^{\dagger}$, single (unmarried/divorced/widowed).

Table 4 Logistic regressions analysis for occurrence of peristomal dermatitis

\begin{tabular}{lccc}
\hline Characteristic & OR & $95 \% \mathrm{Cl}$ & P value \\
\hline Gender & 1.571 & $0.960-2.571$ & 0.072 \\
Diabetes & 3.732 & $1.890-7.367$ & 0.001 \\
Stoma Class & 0.458 & $0.258-0.815$ & 0.008 \\
\hline
\end{tabular}

after stoma education than that not attending the class $(\mathrm{P}=0.012)$. However, there was no significant difference in those who were older than 60 years old, which suggested that the stoma education class had no significant positive effect on the incidence of peristomal dermatitis in elderly patients. This may be related to the decline of the elderly patients' ability to accept centralized health education, so we should explore a more suitable health education model for elderly patients. On one hand, we can provide more opportunities of skill training for elderly patients and their family members. On the other hand, more suitable products should be developed according to the unique characteristic of elderly patients. 
It was reported that diabetes mellitus may be an independent risk factor for peristomal skin complications. In our study, the results of multivariate analysis suggested that diabetes mellitus was a risk factor for early postoperative peristomal dermatitis in patients with ileostomy $(\mathrm{OR}=3.732$, $\mathrm{P}=0.001)$. Patients with stoma should actively treat diabetes and other basic diseases. In our stratified analysis, for patients without diabetes, the rates of peristomal dermatitis was significantly lower in those attending the education class than in those who did not $(\mathrm{P}=0.026)$, while there was no significant difference among those with diabetes.

In addition, self-care patients were reported to be happier and to experience higher health-related quality of life, which inevitably results in a reduction of health care costs (28). Our stratified analysis found that for different caregivers, stoma class was significant indicators of peristomal dermatitis in patients who received stoma care from others $(\mathrm{P}=0.014)$ while not in patients who practiced of self-care $(\mathrm{P}=0.503)$. We supposed that ostomates who practiced self-care paid more attention to ward stomacare practice and ostomates cared for by others and who attended stoma education class received more effective support from their caregivers. Therefore, we should take actions to improve the nursing level for the self-care patients, especial elderly patients. Maybe we can provide teaching video and technique support during follow-up.

Several limitations need to be noted regarding the present study. First, the content was follow-up data by clinics and telephone, and the data may deviate from the actual situation, and the frequency and severity of peristomal dermatitis were not classified. What's more, only $32.99 \%$ of ostomates in our study participated in the stoma education class, which was largely related to the 2 -week interval between stoma education class and the failure of most of patients to participate in stoma education class during their hospitalization. A more effective mode and frequency of centralized health education according to the actual situation should be explored in the near future.

\section{Conclusions}

Regardless of education level, a postoperative stoma education class significantly decreased the rate of peristomal dermatitis in the early postoperative period in CRC patients with ileostomy, especially for those who were younger than 60 years, who received stoma care from others and who were without diabetes.

\section{Acknowledgments}

Funding: This research did not receive any specific grant from funding agencies in the public, commercial, or notfor-profit sectors.

\section{Footnote}

Reporting Checklist: The authors have completed the STROBE reporting checklist. Available at http://dx.doi. org/10.21037/tcr-20-3267

Data Sharing Statement: Available at http://dx.doi. org/10.21037/tcr-20-3267

Conflicts of Interest: All authors have completed the ICMJE uniform disclosure form (available at http://dx.doi. org/10.21037/tcr-20-3267). The authors have no conflicts of interest to declare.

Ethical Statement: The authors are accountable for all aspects of the work in ensuring that questions related to the accuracy or integrity of any part of the work are appropriately investigated and resolved. The study was conducted in accordance with the Declaration of Helsinki (as revised in 2013). The study was approved by the Ethics Committee of the Sixth Affiliated Hospital of Sun Yat-sen University (NO.2020ZSLYEC-040). Informed consent is not needed in this observational and retrospective study.

Open Access Statement: This is an Open Access article distributed in accordance with the Creative Commons Attribution-NonCommercial-NoDerivs 4.0 International License (CC BY-NC-ND 4.0), which permits the noncommercial replication and distribution of the article with the strict proviso that no changes or edits are made and the original work is properly cited (including links to both the formal publication through the relevant DOI and the license). See: https://creativecommons.org/licenses/by-nc-nd/4.0/.

\section{References}

1. Siegel RL, Miller KD, Jemal A. Cancer statistics, 2019. CA Cancer J Clin 2019;69:7-34.

2. Matthiessen P, Hallböök O, Rutegård J, et al. Defunctioning stoma reduces symptomatic anastomotic leakage after low anterior resection of the rectum for cancer: a randomized multicenter trial. Ann Surg 
2007;246:207-14.

3. Tsujinaka S, Tan KY, Miyakura Y, et al. Current Management of Intestinal Stomas and Their Complications. J Anus Rectum Colon 2020;4:25-33.

4. Brown H, Randle J. Living with a stoma: a review of the literature. J Clin Nurs 2005;14:74-81.

5. Danielsen AK, Burcharth J, Rosenberg J. Spouses of patients with a stoma lack information and support and are restricted in their social and sexual life: a systematic review. Int J Colorectal Dis 2013;28:1603-12.

6. van Loon YT, Clermonts SHEM, Belt R, et al. Implementation of an easy in-hospital educational stoma pathway results in decrease of home nursing care services after discharge. Colorectal Dis 2020;22:1175-83.

7. Hughes MJ, Cunningham W, Yalamarthi S. The effect of preoperative stoma training for patients undergoing colorectal surgery in an enhanced recovery programme. Ann R Coll Surg Engl 2020;102:180-4.

8. Faury S, Koleck M, Foucaud J, et al. Patient education interventions for colorectal cancer patients with stoma: A systematic review. Patient Educ Couns 2017;100:1807-19.

9. Persson E, Berndtsson I, Carlsson E, et al. Stomarelated complications and stoma size - a 2-year follow up. Colorectal Dis 2010;12:971-6.

10. Hignett S, Parmar CD, Lewis $W$, et al. Ileostomy formation does not prolong hospital length of stay after open anterior resection when performed within an enhanced recovery programme. Colorectal Dis 2011;13:1180-3.

11. Stokes AL, Tice $S$, Follett $S$, et al. Institution of a Preoperative Stoma Education Group Class Decreases Rate of Peristomal Complications in New Stoma Patients. J Wound Ostomy Continence Nurs 2017;44:363-7.

12. Forsmo HM, Pfeffer F, Rasdal A, et al. Pre- and postoperative stoma education and guidance within an enhanced recovery after surgery (ERAS) programme reduces length of hospital stay in colorectal surgery. Int J Surg 2016;36:121-6.

13. Cressey BD, Belum VR, Scheinman P, et al. Stoma care products represent a common and previously underreported source of peristomal contact dermatitis. Contact Dermatitis 2017;76:27-33.

14. Agarwal S, Ehrlich A. Stoma dermatitis: prevalent but often overlooked. Dermatitis 2010;21:138-47.

15. Salvadalena G. Incidence of complications of the stoma and peristomal skin among individuals with colostomy, ileostomy, and urostomy: a systematic review. J Wound Ostomy Continence Nurs 2008;35:596-607.

16. Park JJ, Del Pino A, Orsay CP, et al. Stoma complications: the Cook County Hospital experience. Dis Colon Rectum
1999;42:1575-80.

17. Nastro P, Knowles CH, McGrath A, et al. Complications of intestinal stomas. Br J Surg 2010;97:1885-9.

18. Murken DR, Bleier JIS. Ostomy-Related Complications. Clin Colon Rectal Surg 2019;32:176-82.

19. Meisner S, Lehur PA, Moran B, et al. Peristomal skin complications are common, expensive, and difficult to manage: a population based cost modeling study. PloS One 2012;7:e37813.

20. Thorpe G, McArthur M, Richardson B. Healthcare experiences of patients following faecal output stomaforming surgery: a qualitative exploration. Int J Nurs Stud 2014;51:379-89.

21. Steinhagen E, Colwell J, Cannon LM. Intestinal Stomas-Postoperative Stoma Care and Peristomal Skin Complications. Clin Colon Rectal Surg 2017;30:184-92.

22. Aarts MA, Rotstein OD, Pearsall EA, et al. Postoperative ERAS Interventions Have the Greatest Impact on Optimal Recovery: Experience With Implementation of ERAS Across Multiple Hospitals. Ann Surg 2018;267:992-7.

23. Wahl TS, Goss LE, Morris MS, et al. Enhanced Recovery After Surgery (ERAS) Eliminates Racial Disparities in Postoperative Length of Stay After Colorectal Surgery. Ann Surg 2018;268:1026-35.

24. Altuntas YE, Kement M, Gezen C, et al. The role of group education on quality of life in patients with a stoma. Eur J Cancer Care (Engl) 2012;21:776-81.

25. Seo HW. Effects of the frequency of ostomy management reinforcement education on self-care knowledge, selfefficacy, and ability of stoma appliance change among Korean hospitalised ostomates. Int Wound J 2019;16 Suppl 1:21-8.

26. Koc U, Karaman K, Gomceli I, et al. A Retrospective Analysis of Factors Affecting Early Stoma Complications. Ostomy Wound Manage 2017;63:28-32.

27. Nybaek H, Bang Knudsen D, Norgaard Laursen T, et al. Skin problems in ostomy patients: a case-control study of risk factors. Acta Derm Venereol 2009;89:64-7.

28. Zhang Y, Xian H, Yang Y, et al. Relationship between psychosocial adaptation and health-related quality of life of patients with stoma: A descriptive, cross-sectional study. J Clin Nurs 2019;28:2880-8.

Cite this article as: He D, Liang W, Yao Q, Zhao J, Liu R, Chen G, Wang H, Ye X, Huang R. The effect of stoma education class on peristomal dermatitis in colorectal cancer patients with defunctioning ileostomy-a retrospective study of 491 patients. Transl Cancer Res 2021;10(2):581-588. doi: 10.21037/tcr-20-3267 